\title{
Lactobacillus Paracasei R3 Protects Against Dextran Sulfate Sodium (DSS)- Induced Colitis in Mice via Regulating Th17/Treg Cell Balance
}

Juan Huang

Guangdong Medical University https://orcid.org/0000-0002-7121-1900

Ziyan Yang

Guangdong Medical University

Yanyun Li

Guangdong Medical University

Xingxing Chai

Guangdong Medical University

Yanfang Liang

Binhaiwan Central Hospital of Dongguan

Bihua Lin

Guangdong Medical University

Ziyu Ye

Guangdong Medical University

Shaobing Zhang

Guangdong Medical University

Zhengping Che

Guangdong Medical University

Hailiang Zhang

Guangdong Medical University

Xueying Zhang

Guangdong Medical University

Zhao Zhang

Guangdong Medical University

Tao Chen

Guangdong Medical University

Weiqing Yang

Guangdong Medical University

Jincheng Zeng ( $\nabla$ zengjc@gdmu.edu.cn)

Guangdong Medical University

\section{Research Article}

Keywords: Lactobacillus paracasei, colitis, dextran sulfate sodium, Treg, Th17, whole genome sequencing

Posted Date: April 16th, 2021

DOI: https://doi.org/10.21203/rs.3.rs-405110/v1

License: (1) (1) This work is licensed under a Creative Commons Attribution 4.0 International License. Read Full License

Version of Record: A version of this preprint was published at Journal of Translational Medicine on August 18th, 2021. See the published version at https://doi.org/10.1186/s12967-021-02943-x. 


\begin{abstract}
Inflammatory bowel diseases (IBD), mainly comprising ulcerative colitis (UC) and Crohn's Disease, are most often a polygenic disorder with contributions from the intestinal microbiome, defects in barrier function, and dysregulated host responses to microbial stimulation. Strategies that target the microbiota have emerged as potential therapies and, of these, probiotics have gained the greatest attention. Herein, we isolated a strain of Lactobacillus paracasei R3 (L.p R3) with strong biofilm formation ability from infant feces. Interestingly, we also found L.p R3 strain can ameliorate the general symptoms of murine colitis, alleviate inflammatory cell infiltration and inhibit Th17 while promote Treg function in murine DSS-induced colitis. Overall, this study suggested that $L . p$ R3 strain significantly improves the symptoms and the pathological damage of mice with colitis and influences the immune function by regulating Th17/Treg cell balance in DSS-induced colitis in mice.
\end{abstract}

\title{
1 Introduction
}

Inflammatory bowel diseases (IBD), mainly comprising ulcerative colitis (UC) and crohn's disease, are most often a polygenic disorder with contributions from the intestinal microbiome, defects in barrier function, and dysregulated host responses to microbial stimulation. Strategies that target the microbiota have emerged as potential therapies for IBD and, of these, probiotics have gained the greatest attention.

From discovery to clinical application, probiotics have been developed and explored for many years. The category and mechanism of probiotics are gradually clear, and the clinical application is more and more extensive, and their functions mainly include intestinal protection, immune regulation, antibacterial effect, nutrition, anti-tumor, protecting liver, reducing blood lipid and so on[1-7]. Intestinal probiotics, as a kind of active microorganisms, need to be colonized in human intestinal epithelial cells, and then communicate with the intestinal mucosa layer to play an important role in the metabolic, immune and intestinal health protection function of host individuals[1].

Supplementation of probiotics can be used as an treatment IBD. However, due to the complex pathogenesis of IBD and the influence of individual probiotics, there are many probiotics in the literature, but few strains are used for actual transformation. Therefore, it is still necessary to provide scientific and detailed evidence for the beneficial effects of probiotics. Escherichia coli Nissle 1917 is a non-pathogenic probiotic that can relieve clinical symptoms in patients with UC with the same efficacy and safety as mesalazine (MSLZ)[8-10]. The synergistic effect of probiotics and 5-ASA in patients with mild to moderate UC promotes clinical remission. Studies have shown that probiotics could reduce proinflammatory cytokines (such as TNF-a and IL-1 $\beta$ ) and increase antiinflammatory factors(IL-10) via inhibiting TLR4/NF-KB signaling pathway and PI3K/Akt/NF-KB signaling pathway to maintaining UC remission[11].

Administration of Lactobacillus paracasei strains also improves immunomodulation and changes the composition of gut microbiota leading to improvement of colitis in mice[12, 13]. Herein, we isolated a strain of Lactobacillus paracasei R3 (L.p R3) with strong biofilm formation ability from infant feces.

Interestingly, we also found L.p R3 strain can ameliorate the general symptoms of murine colitis, alleviate inflammatory cell infiltration and inhibit Th17 while promote Treg function in murine DSS-induced colitis.

\section{Materials And Methods}

\subsection{Probiotics isolation}

L.p R3 were isolated from baby feces and identified by API 50CH strips (BioMerieux) and 16S rDNA sequence in our laboratory. The strain was grown in Man, Rogosa, Sharpe (MRS) broth (Oxoid, Cambridge, UK) and on MRS agar (Oxoid,Cambridge, UK). Inoculated plates were incubated for $48 \mathrm{~h}$ at $35^{\circ} \mathrm{Cunder}$ anaerobic conditions. L.p R3 was incubated overnight at $35^{\circ} \mathrm{C}$ in MRS broth.

\subsection{Detection of growth curve}

Preparation of fresh bacterial suspensions for growth curves, gastrointestinal tolerance and biofilm formation experiments. MRS broth was used to activate L.p R3 strain, strain cells were collected by centrifugation at $4,000 \mathrm{~g} \times \mathrm{g}$ for $10 \mathrm{~min}$, washed twice with PBS, and resuspended at $1.5 \times 10^{8} \mathrm{cfu} / \mathrm{ml}$. The fresh suspension was inoculated into $200 \mathrm{ml}$ MRS liquid medium according to $2 \%\left(\mathrm{v} / \mathrm{v}\right.$ )inoculation and $35^{\circ} \mathrm{Cculture}$ for $24 \mathrm{~h}$. From $0 \mathrm{~h}$ to $48 \mathrm{~h}$, sampling every $2 \mathrm{~h}$, measuring the $\mathrm{OD}_{630}$ value. Each time point is repeated 3 times, taking the average value. MRS broth was a blank control. And the growth curve was drawn with culture time as horizontal coordinate and absorbance as vertical coordinate.

\subsection{Gastrointestinal fluid tolerance}

Equipped with artificial intestinal fluid and $\mathrm{PH}=2,3,4,7$ gastric juice. The fresh suspension was inoculated into $20 \mathrm{ml}$ MRS liquid medium according to $2 \%$ $(\mathrm{v} / \mathrm{v})$ inoculation and $35^{\circ} \mathrm{C}$ culture for $16 \mathrm{~h}$. Cultures were collected by centrifugation at $4,000 \mathrm{~g} \times \mathrm{g}$ for $10 \mathrm{~min}$, washed twice with PBS, discard supernatant. First, the bacterial cells were treated with 4 kinds of gastric juice, $35^{\circ} \mathrm{C}$ and $50 \mathrm{r} / \mathrm{min}$, for $3 \mathrm{~h}$,count the number of live bacteria at $30 \mathrm{~min}, 90 \mathrm{~min}$ and $180 \mathrm{~min}$ time point. After 3 hours of gastric juice treatment, centrifuge to discard gastric juice, then add the same volume of intestinal fluid, $35^{\circ} \mathrm{C}$ and $50 \mathrm{r} / \mathrm{min}$, for $3 \mathrm{~h}$, count the number of live bacteria at $270 \mathrm{~min}$ and $360 \mathrm{~min}$ time point. The concentration of bacterium was determined by colony plate counting method. Each group was set up 3 parallel and repeated 3 times. Survival rate $=\left(\right.$ number of live bacteria per time point $\left.(\mathrm{cfu} / \mathrm{ml}) / 1.5 \times 10^{8} \mathrm{cfu} / \mathrm{ml}\right) \times 100 \%$.

\subsection{Biofilm formation}

Semi-quantitative determination of biofilm formation ability of strain by micro-pore plate. The fresh suspension was inoculated into $200 \mathrm{ml}$ MRS liquid medium according to $2 \%(\mathrm{v} / \mathrm{v})$ inoculation, mix well, add to 96 hole plate, each hole $200 \mathrm{ul}, 35^{\circ} \mathrm{Cstatic}$ culture for 48 h, determine the $\mathrm{OD}_{630}$ value by enzyme labeling instrument, recorded as A1, MRS broth was a blank control.Discard excess medium, washed twice with PBS to remove residual media and surface plankton bacteria, with 200 uLmethanol fixed for $10 \mathrm{~min}$ in each hole, natural drying, with $100 \mathrm{ul} 1 \%$ crystal violet solution to dye 30 min, washed twice with PBS 
to remove excess dyes. When dried naturally, $200 \mathrm{ul} 95 \%$ of ethanol were added to each hole to dissolve the crystal violet completely. The $\mathrm{OD}_{630}$ value was measured, recorded as A2. Each group was set up 3 parallel and repeated 3 times. The adhesion rate is used to express the film forming ability, recorded as $B$, $B=A 2-A 2 C / A 1-A 1 C, A 1 C$ and $A 2 C$ are the blank control absorbance value when measuring $A 1$ and $A 2$, respectively. The $B<0.1$ is non-adhesion, $0.1 \leqq B<0.5$ low-level film formation, $0.5 \leqq B<1$ medium-level film formation and $B \geqq 1$ strong adhesion film formation.

\section{5 whole genome sequencing analysis}

Sequencing and bioinformatics analysis commissioned by Guangdong Nanxin Medical Technology Co., Ltd. Using a Illumina platform, the operation is mainly divided into five steps: extraction of genome DNA, library preparation, high-throughput sequencing, genome assembly, genome fine mapping and biological information analysis (including three aspects of gene prediction, gene annotation and genome comparative analysis). In gene function annotation analysis, a total of 11 databases were used, including ARDB, CARD, CAZY, GO, COG, KEGG, T3SS, NR,DBCAN, SWISSPROT and IPR .

\subsection{Safety evaluation of L.p R3 strain}

Acute toxicity test: 12 Balb/c mice, after 1 week of adaptive feeding, randomly divided into 2 groups including control group and $L . p$ R3 group,six mice per group. MRS broth was used to activate L.p R3 strain, strain cells were collected by centrifugation at 4,000g $\times \mathrm{g}$ for 10 min,washed twice with PBS, and resuspended at $1 \times 10^{12} \mathrm{cfu} / \mathrm{ml}$. The experimental group was given $200 \mathrm{ul}$ bacteria suspension each time, and the control group was given $200 \mathrm{ul}$ saline each time, once a day, for 30 days. Record the general signs of the mice daily, such as movement, hair, weight, death or not. On the 31 st day, Cervical dislocation method was used to kill and dissect mice to take their organs. To observe the morphological changes of heart, liver, spleen, lung and kidney, and to measure organ quality, Calculate organ index $=\mathrm{m} 1 / \mathrm{m} 2(\mathrm{~m} 1$ is organ quality $(\mathrm{g}), \mathrm{m} 2$ was weight quality in mice $(\mathrm{g})$ ).

\subsection{Design of animal experiments}

Six-week-old C57BL/6 mice(weighing $20.0+2.0 \mathrm{~g}$, from Guangdong Medical Laboratory Animal Center, Foshan, Guangdong Province, China) were housed in plastic cages (at $22 \pm 2^{\circ} \mathrm{C}$ and $50 \sim 60 \%$ relative humidity with a $12 \mathrm{~h}$ dark $/ 12 \mathrm{~h}$ light cycle) with free access to diet and water. Acute colitis was induced by administering 3\% (w/v) DSS (molecular mass 36,000-50,000 Da; MP Biochemicals) in drinking water. The concentrations of L.p R3 strain and mesalazine (MSLZ) used in the experiment were $1.0 \times 10^{9} \mathrm{CFU} / \mathrm{mL}$ and $52 \mathrm{mg} / \mathrm{mL}$, respectively. $\mathrm{C} 57 \mathrm{BL} / 6$ mice were randomly divided into five groups $(\mathrm{n}=6)$, including Normal group, DSS group,MSLZ group,L.p R3 group and L.p R3 combined with MSLZ group(L.p R3 + MSLZ group). For 14 days, each mouse was given 200 $\mathrm{uL} / \mathrm{d}$ by gavage at a fixed daily time. From 8-14 days, the normal group was free to drink double distilled water, and the other 4 groups of mice were free to drink 3\% DSS solution for 7 days to establish an acute UC model. Observe and record the daily condition of mice during the test. After the experiment, serum and colon samples from mice were collected for study. The experiment was approved by and performed in accordance with the guidelines of the ethics committee of Guangdong Medical University.

\subsection{Determination of disease activity index(DAl)}

The disease activity index (DAl) was determined by scoring the degree of weight loss, stool stiffness, and the presence of occult or whole blood (Table 1). Occult blood in faecals was evaluated with a Faecal Occult Blood Test Kit (Jiancheng Biotech, Nanjing,china P.R).

Table 1

Disease activity index score

\begin{tabular}{|llll|}
\hline Score & Weight loss & Stool cinsistency & Gross bleeding \\
\hline 0 & None & Normal & negative \\
\hline 1 & $1-5 \%$ & & \\
2 & $6-10 \%$ & Losse stool & Haemoccult \\
\hline 3 & $11-15 \%$ & & Bleeding(visible) \\
\hline 4 & $>15 \%$ & Diarrhea & \\
\hline \multicolumn{2}{|l|}{ The disease activity index=(combined score of weight loss, stool consistency and bleeding)/3. } \\
\hline
\end{tabular}

\subsection{Histological assessment}

Colon length was measured as described above to assess colon shortening. $1 \mathrm{~cm}$ distal colon was fixed with PBS-buffered $10 \%(\mathrm{v} / \mathrm{v})$ formalin buffer for 48 hours, then embedded in paraffin, cut into 5-um thick sections, hematoxylin and eosin (H\&E)Staining and inspection with light microscope. A blinded histopathologic analysis was used to score the stage of colitis based on morphological criteria Ringed (Table 2). Randomly select 3 sections of each sample and examine 10 random fields (magnification 100x) in each section. The average value for each part is calculated by the average level. 
Table 2

Histological disease scoring system

\begin{tabular}{|ll|}
\hline Score & Histological features \\
\hline 0 & Normal colonic mucosa \\
\hline 1 & Loss of one-third of the crypts \\
\hline 3 & Loss of two-third of the crypts \\
& $\begin{array}{l}\text { The lamina propria is covered with a single lay } \\
4\end{array}$ \\
\hline
\end{tabular}

\subsection{Immunohistochemistry}

Immunohistochemistry was performed on 4 um thick paraffinembedded sections of the colon. The with incubated were sections anti-FOXP3(1:200), antiCD25(1:200), anti-IL-17A(1:20 0) at $4^{\circ} \mathrm{C}$ overnight.VECTASTAIN ABC Kit (Vector, CA)was used as secondary antibody. Immunoreactivity was analyzed with a microscope (Olympus AX70, Olympus Optical Corp., Tokyo, Japan). The total number of CD25, FOXP3, IL-17A and IL-10 positve cells in each of four nonadjacent fields of view was counted per colon sample(magnification, 100x).

\subsection{Western blot}

The tissue was homogenized with lysis buffer, an equal amount of protein (50 ug) was analyzed by $12 \%$ SDS-polyacrylamide gel electrophoresis, and the blotted membrane was blocked with 5\% BSA in TBST for 60 mins at room temperature, anti-FOXP3 (1:1000, Abcam), anti-CD25 (1:1000, Affinity Biosciences), anti-IL-17A (1:1000, Affinity Biosciences), anti-L-10 (1:1000, bs-6761R, Bioss) and FN-y (1:1000, Affinity Biosciences) antibody overnight at 4C.GAPDH (1:1000.Sigma) was used as a loading control. The HRP-conjugated anti-rabbit or anti-mouse IgG(1:2000, Cell Signaling) was used as a secondary antibody and the membrane was developed using an enhanced chemiluminescence system(Amersham Pharmacia Biotech).

\subsection{Measurement of cytokine}

Cytokines such as IL-10, IFN-y, IL-17A and IL-17F were measured in duplicate with Multi-Analyte Flow Assay Kit(Biolegend).

\subsection{Statistical analysis}

Figures and statistics were performed using Prism 7.0 a software (Graphpad Inc.). All data represent mean \pm SEM. Data that was not successfully distributed was log converted and evaluated for distribution. Using Student's t-test or ANOVA with Tukey test to determine significant differences between two groups or analyze significant differences between multiple tests Group.

\section{Results}

\subsection{Biological properties of L.p R3}

L.p R3 were isolated from infant feces and identified by API 50CH Biochemical reaction system, named and preserved in our laboratory. The dynamic growth curve in vitro showed that L.p R3 strain had a logarithmic phase at $4 \mathrm{~h} \sim 18 \mathrm{~h}$ and then entered the stationary phase, no apparent death phase (Fig. 1A). Under successive effects of artificial gastric and intestinal fluids, the survival rate of L.p R3 was above $90 \%$ and $70 \%$ in PH=3.0, 4.0 and 7.0 gastric and intestinal fluids, respectively. The survival rate of $L . p$ R3 reached $(58.76 \pm 1.32) \%$ and $(43.08 \pm 0.27) \%$ after $3 \mathrm{~h}$ in PH 2.0 artificial gastric juice and intestinal fluids treatment(Fig. 1B). These results indicate that L.p R3 has good tolerance to gastrointestinal fluid and can survive and maintain activity under adverse gastrointestinal conditions. Biofilm formation rate of L.p R3 strain was more than 0.6 in the experiment of semi-quantitative determination of biofilm formation ability, showed that the strain had better ability of biofilm formation(Fig. 1C口D).

\subsection{Whole genome sequencing analysis of L.p R3}

To look for other possible characteristics of L.p R3, its entire genome was sequenced(Fig. 2A). The main features of $L . p$ R3 genome are reported in Table 3.The assembling of reads generated 92 contigs, giving a genome size of $3.095 \mathrm{Mb}$ with a GC content of $46.17 \%$. Using multiple databases to analyze the gene function of the strain, gene prediction and annotation indicated the total number of 3171 predicted protein-coding sequences (CDSs) and showed 60 structural RNAs. The strain had no plasmids on its chromosomes and no virulence genes were detected. It is noteworthy that the strain genes involve antibiotic resistance genes and TXSS systems associated with pathogenic mechanisms of Gram-negative bacteria. However, the similarities between the detected resistance gene locus and the corresponding antibiotic resistance gene sequences were less than $80 \%$. And its did not evidence any acquired resistance gene in L.p R3, hence this strain can be considered safe regarding possible transmissible antibiotic resistances. As for the existence of TXSS system, whether the strain has the risk of infection needs further study. COG database annotations were shown in Fig. 2B. The largest part of this subsystem is allocated to the Carbohydrate transport and metabolism(13.01\%), General function prediction only(9.32\%), Translation, ribosomal structure and biogenesis(9.28\%), Amino acid transport and metabolism(8.90\%),Transcription(6.48\%), Inorganic ion transport and metabolism(5.16\%) and Cell wall/membrane/envelope biogenesis(5.06\%), respectively. It is noteworthy that the strain contains proteins related to Lipid transport and metabolism(3.27\%) and Mobilome: prophages, transposons (1.66\%). 
Table 3

Genome feature of L.p R3

\begin{tabular}{|l|l|}
\hline Attribute & Values \\
\hline Genome size & $3,095,229 \mathrm{bp}$ \\
\hline G + C content (\%) & 46.17 \\
\hline Contig N50 & $89,515 \mathrm{bp}$ \\
\hline Contig L50 & 11 \\
\hline Number of contigs & 92 \\
\hline Number of protein conding sequence (CDSs) & 3,171 \\
\hline Number of rRNAs & 3 \\
\hline Number of tRNAs & 57 \\
\hline
\end{tabular}

\subsection{Safety evaluation of L.p R3}

L.p R3 did not produce hemolysis using colombian blood agar plate by anaerobic culture for $48 \mathrm{~h}$. By feeding L.p R3(concentration of $1 \times 10^{12}$ cfu/ml) to mice for 7day and 30 day, there was no significant difference in body weight, organ index, and blood glucose compared with the control group (Fig. 3). Experimental results showed that the activity and mental state of mice were normal, eating and drinking water were stable, the fur was bright, the fecal state was normal, no death occurred and no lesions were found in Heart, liver, spleen, lung and kidney organ. And Body weight and major organ index of mice were not significantly different compared with the control group $(p>0.05)$ (Fig. 3 ). The results suggest that the strain had no risk of infection and did not affected the normal physiology of mice.

\subsection{L.p R3 strain ameliorates the general symptoms of murine colitis}

In DSS-induced UC model, normal group mice showed normal activity, mental state, normal defecation,bright hair and normal weight gain. And other four groups mice began to develop different degrees of UC symptoms on the third day of free drinking of $3 \%$ DSS solution. These results show that the model is successful. Especially, the symptoms of mice in the DSS group are becoming more and more serious, with poor spirit, obvious blood stool and hair removal symptoms. Compared with the treatment group,the weight ratio of the DSS group decreased significantly $(p<0.05)(F i g .4 A)$. The DAI score, which is an indicator of the severity of colitis, including the extent of body weight loss, haemoccult positivity or gross bleeding and stool consistency, was evaluated for each animal (Table 1). As shown in Fig. 4B, according to normal group, the order of DAl score from large to small is DSS group > MSLZ group $\geq$ LP R3 group > LP R3 + MSLZ group. DSS group began to show significantly higher DAl values compared to the control group $(P<0.05)$. The DAI values of MSLZ + Lp R3 groups were significantly lower $(p<0.05)$ than DSS group.The results suggesting that L.p R3 can alleviate weight loss and UC symptoms in mice caused by DSS.

\subsection{L.p R3 strain alleviates inflammatory cell infiltration in murine DSS-induced colitis}

We studied the colon of experimental mice and the results were shown in Fig. 4C and 4E. During colonic inflammation, the length of the mouse colon is shortened by ulceration. This is accompanied by adhesions of colonic tissue. In DSS group, colon length was significantly shorter than in the control group ( $p$ $<0.05)$. The colon length was recovered to different degrees in the three treated-groups compared with DSS group, and the recovery was most obvious in LP R3 + MSLZ group $(p<0.001)$. In further colon histopathological experiments, mice colonic structure damage, glands incomplete, a large number of inflammatory cell infiltration, typical inflammatory changes in DSS group. And the histological injury score of DSS group was significantly higher than that of other 4 groups $(p<0.001)$. while the L.p R3 administered mice exhibited significantly less histological damage.There was less loss of crypt and goblet cells and less infiltration of various immune cells in the L.pR3 administered mice(Fig. 4E). Similarly,the systemic inflammatory response, assessed by splenomegaly, is shown in Fig. 4D. Spleen index were also significantly higher in DSS mice than in controls $(p<0.05)$. In mice fed with $L$. $p$ R3, the mean spleen index was lower than in DSS animals $(p<0.05)$ and more similar to control animals. The results confirmed that the strain can relieve inflammation and pathological damage of the colon by DSS-induced.

\subsection{L.p R3 strain reverses inflammatory cytokines IL-17A/IL-10 rates}

Determination immune factors of IFN-y, IL-10, IL-17A and IL-17F in peripheral serum of mice by liquid-phase chip method, to explore the immunomodulatory mechanism of L.p R3 strain. As shown in the Fig. 5, four cytokines have different levels in peripheral blood of each groups. Normal group as reference,the contents of IFN-y, IL-17A, IL-17F and IL-10 were increased in DSS group, except for IL-10, there were significant differences( $<<0.05)$. Interestingly, the three treatment groups were just the opposite of the model group.The levels of peripheral blood IFN-y, IL-17A and IL-17F decreased significantly and IL-10 increased significantly in three treatment groups $(p<0.05)$. Moreover, the IL-17A/IL-10 value of NS group was set to 1 , and the IL-17A/IL-10 values of each group were compared to evaluate the changes of Th17/Tregs cell balance in mice after treatment. The results showed that the IL-17A/IL-10 value of DSS group was significantly higher than that of the other four groups $(\mathrm{p}<0.05)$. This indicates that Th17 cells infiltrate and secrete the pro-inflammatory factor IL-17A during inflammation. After treatment with L.p R3 or/and MSLZ, the anti-inflammatory factor IL-10 increases and the IL-17A/IL-10 value reverses.

\subsection{L.p R3 strain inhibits Th17 and promotes Treg function in murine DSS-induced colitis}


Th17 cell is a T cell subset that can secrete IL-17. Tregs cells are characterized by the expression of Foxp3, CD25 and CD4. Therefore, the expression of cells in tissues can be reflected by detecting Th17 and Tregs related cell markers. The expression of Foxp3, IL-10, IL-17A and IFN-y in colonic tissues of mice in each group was detected by immunohistochemistry as shown in Fig. 6A and 6B. Normal group as reference, the expression of Foxp3, IL-10, IL-17A and IFN- $y$ increased to varying degrees in DSS group and 3 treated groups. And compared with DSS group, in the treatment groups of MSLZ, LP R3 and LP R3 + MSLZ, Foxp3 and IL-10 expression increased in turn, while IL-17A and IFN- $y$ expression decreased in turn, they were statistically different ( $p<0.05)$. The expression of CD4, CD25, Foxp3, IL-10, IL-17A and IFN- $y$ in colon of mice in each group was further examined by Western blotting as shown in Fig. 6C. Normal group as reference,the CD4, CD25 and Foxp3 expression of the three treatment groups was higher than that of the DSS group, and the differences were statistically significant $(p<0.05)$. And the expression of IL-17A and IFN-y in treatment group was significantly lower than that in DSS group $(p<0.05)$. IL-10 not well detected, probably because protein expression is affected by a variety of factors. The above results suggest $L$. $p$ R3 could promote the expression of Tregs cell markers and inhibits the expression of Th17 cell markers. Treg/Th17 cell imbalance in inflammation caused by DSS was restored after treatment.

\section{Discussion}

L.p R3 strain, as a member of traditional probiotics, was isolated from infant faeces by our team. We studied the survival ability of the strain in vitro. The dynamic growth curve in vitro showed that $L . p$ R3 strain had a logarithmic phase at $4 \mathrm{~h} \sim 18 \mathrm{~h}$ and then entered the stationary phase, no apparent death phase.The strain had good characteristics with gastrointestinal fluid tolerance and strong biofilm formation ability. Probiotics are often used as active microorganisms, which need to withstand the persecution of gastrointestinal fluid and reach intestinal colonization with a high number of live bacteria, and to play a role in promoting the health of the body. Colonization is the premise of growth and development of probiotics and a physiological function under adhesion. The colonized bacteria can form biofilm on the surface of adhesion membrane to prevent the invasion of foreign bacteria and protect the health of intestinal mucosa. Therefore, acid tolerance, bile salt tolerance and intestinal mucosal adhesion all used as criteria for screening probiotics[14].

L.p as an important member of Lactobacillus, is Gram-positive bacterium widely found in human oral cavity, intestinal tract and fermented foods. L.p has the function of regulating intestinal flora balance, enhancing human immunity and anti-tumor[15-17]. At present, it has been used in food, health care and industrial production, which has attracted wide attention at home and abroad. Lactobacillus paracasei subsp. paracasei NTU 101 (NTU 101$)$ is a multifunctional strain that has been shown in previous studies to possess anti-inflammatory properties and to exert a modulatory effect on intestinal bacteria associated with certain pathogenic mechanisms of IBD. The probiotic strains could reduce oxidative stress and the inflammatory response in DSS-treated mice by enhancing the antioxidative capacity of total antioxidants capacity (GR, GSH, CAT, SOD, MDA), and hindering the secretion of proin-inflammatory cytokines (such as TNF-a, IL-6, IFN-y, and IL-12) [12]. Lactobacillus paracasei ssp. paracasei YBJ01 reduced d-galactose-induced oxidation in male Kuming mice. Strain LPSP-YBJ01 significantly increased serum superoxide dismutase (SOD), glutathione peroxidase, and total-antioxidant capability, and inhibited generation of malondialdehyde in a dose-dependent manner[18]. In addition, strain LPSP-YBJ01 also increased the hepatic and splenic protein expressions of some antioxidant enzymes such as catalase, Cu/Zn-SOD, and Mn-SOD in mice treated with d-galactose[18].

The safety of probiotic strains is also important, including potential pathogenic virulence factors and resistance. At present, the probiotic products in China only list the name of bacteria, there is no specific strain name and related genetic background information, but the probiotic and safety of probiotics are strain specific. Genomics studies on the probiotic and safety of probiotics are essential to obtain lactic acid bacteria that can be used to develop probiotics. This method can fully reveal the genetic information of lactic acid bacteria, systematically explain the physiological function and metabolic mechanism of lactic acid bacteria, lay the foundation for the systematic classification and genetic evolution of lactic acid bacteria, and provide the basis for breeding excellent strains.In this study, the whole genome sequencing showed that the genome size of L.pR3 strain is $3.095 \mathrm{Mb}$ with a GC content of $46.17 \%$, and possesses 3171 protein-coding sequences. COG annotation data of L.p R3 shown that the protein coding sequence mainly involves the basic physiological activities of the strain,such as Carbohydrate transport and metabolism(13.01\%), General function prediction only(9.32\%), Translation, ribosomal structure and biogenesis(9.28\%), Amino acid transport and metabolism(8.90\%), Transcription(6.48\%), Inorganic ion transport and metabolism(5.16\%) and Cell wall/membrane/envelope biogenesis(5.06\%). It is noteworthy that the strain contains proteins related to Lipid transport and metabolism(3.27\%). L.p R3 may have lipid-lowering potential, it is valuable to be researched fartherly. Fortunately,the strain had no plasmids on its chromosomes and no virulence genes were detected. It was safe for antibiotic resistance gene delivery and virulence gene. To further evaluate the safety of L.p R3, we also examined its hemolysis on the blood plate and its infection risk in mice. The strain did not produce hemolytic rings in vitro, nor did it cause infection in mice fed with high concentration of live bacteria, and did not affect mice normal physiological activities.

L.p R3, as a probiotic strain, were specially studied on the function of intestinal protection in our laboratory. The mouse model of ulcerative colitis was successfully induced by DSS. The treatment of UC model mice by intragastric administration of L.p R3 significantly relieved UC symptoms and pathological damage of colon tissue. During our study, UC model mice were significant the body weight loss, poor mental state, hematochezia and hair loss,and their colon pathology showed mucosal tissue damage and extensive inflammatory cell infiltration. Interestingly, the above-mentioned conditions in mice treated with MSLZ or L.p R3 improved significantly. DAl and histological damage scores were used to quantify the extent of damage in each group mice.The values of two indicators are in the same order, and the order from large to small is DSS group > MSLZ group $\geq$ LP R3 group > LP R3 + MSLZ group. This study showed that MSLZ and L.p R3 had a therapeutic effect on UC, and the combination of probiotics and MSLZ had a better therapeutic effect.

UC is a refractory inflammatory disease that mainly affects the intestine, and is one of the chronic diseases that endanger human health. Global prevalence is projected to affect up to 30 million individuals by 2025[19]. The incidence of UC continues to increase, but so far, the specific pathogenesis of UC is unknown. It is generally believed that the pathogenesis of UC involves the results of multiple interactions including genetics, microorganisms, and the body's immune system[20]. In recent years, the pathogenesis of UC has been continuously studied through UC patients and animal models of UC, and it has been found that the occurrence and development of UC are closely related to the balance of Foxp3 + Tregs cells and Th17 cells (producing IL-17A and IL-17F)in vivo[21-23]. Regulatory $\mathrm{T}$ cells(Tregs) are a subset of $\mathrm{T}$ cells with negative immune regulation. Tregs play an important role in maintaining immune balance and forming peripheral immune tolerance. Tregs can participate in the inflammatory response in vitro through a cell contact-dependent mechanism. Tregs can also

Page 6/13 
participate in the pathogenesis of UC by regulating the release of inhibitory cytokines (IL-10, etc.), and their low number or dysfunction can lead to the occurrence of the disease[23-25]. T helper cell 17(Th17) is a subset of T cells capable of secreting IL-17. IL-17 is a pro-inflammatory cytokine with proinflammatory activity that plays diverse roles in different diseases. The level of IL-17A was significantly increased in UC patients compared with normal subjects, and similarly, in a mouse model of ulcerative colitis, the content of IL-17A was also significantly higher[26, 27].

In this study, we found that Tregs and Th17 cells are keep balance under normal conditions. But in the inflammatory state, Th17 cells migrate from the circulatory system to local inflammatory sites in the intestine and are enriched at the site of inflammatory injury, resulting in a highly activated mucosa of the digestive tract, inducing intestinal immune response, increased release of injurious cytokines, leading to mucosal injury, and compensatory increase of Treg cells, but their increased levels cannot counter the increased levels of pro-inflammatory factors, resulting in an inflammatory response. The expression of Th17 and Tregs cell markers in colon tissues was detected by immunohistochemistry and Western blotting. In the results of immunohistochemistry,normal group as reference, the expression of Foxp3, IL-10, IL-17A and IFN- $y$ increased to varying degrees in DSS group and 3 treated groups. Compared with DSS group, L.p R3 had the same effect as MSLZ. The strain increased Foxp3 and IL-10 expression, but decreased IL-17A and IFN-y expression, they were statistically different ( $\mathrm{p}$ $<0.05)$. Again, in the results of Western blotting, the CD4,CD25 and Foxp3 expression of the three treatment groups was higher than that of the DSS group, while the expression of IL-17A and IFN-y in treatment group was significantly lower than that in DSS group $(p<0.05)$. Obviously, L.p R3 could promote the expression of Tregs cell markers and inhibits the expression of Th17 cell markers. the Treg/Th17 cell imbalance in inflammation caused by DSS was restored after treatment.

Detection of cytokines in serum of experimental mice and further analysis of anti-inflammatory mechanism of L.p R3.The contents of IFN-y, IL-17A, IL-17F and IL-10 were increased in DSS group,except for IL-10, there were significant differences $(p<0.05)$. Interestingly, the three treatment groups were just the opposite of the model group. The levels of peripheral blood IFN- $y$, IL-17A and IL-17F decreased significantly and IL-10 increased significantly in three treatment groups $(p<0.05)$. Moreover, the IL-17A/IL-10 value of DSS group was significantly higher than that of the other four groups $(p<0.05)$. This indicates that Th17 cells infiltrate and secrete pro-inflammatory factors IL-17A during inflammation. With L.p R3 or/ and MSLZ treatment, the IL-10 of ant-inflammatory factors increased, the value of IL-17A/IL-10 reversed. To further confirm that L.p R3 has a good therapeutic effect on UC by regulating Th17/Treg cell balance.

Therefore, we speculated that the supplementation of L.p R3 improved the imbalance of intestinal microorganism, down-regulated the activity of Th17 cells, decreased the secretion of pro-inflammatory cytokines and induced the production of Tregs, and achieved a new balance of intestinal pro-inflammatory and anti-inflammatory cytokines, thereby improving intestinal inflammatory response and mucosal injury, which may be one of the important mechanisms of $L . p$ R3 treatment of UC. In this study, the therapeutic effect of L.p R3 was similar to that of mesalazine, and the combination therapy was better, providing a new choice for clinical treatment.

\section{Conclusions}

In summary, L.p R3 significantly improves the symptoms and the pathological damage of mice with colitis and influences the immune function by regulating Treg/Th17 cell balance in DSS-induced colitis in mice. And L.p R3 had the advantages of high safety, good gastrointestinal fluid tolerance and strong biofilm formation ability.These results provide a good basis for commercial production. However, whether the clinical application of $L . p$ R3 bacteria could achieve the same therapeutic effect, considering the complexity between human body and experimental animals, needed further study.

\section{Abbreviations}

L.p

Lactobacillus paracasei; UC:ulcerative colitis; IBD:Inflammatory bowel diseases; DSS:Dextran sulfate sodium; DAl:disease activity index; MSLZ:mesalazine;

\section{Declarations}

\section{Ethics approval and consent to participate}

This study was approved by and performed in accordance with the guidelines of the ethics committee of Guangdong Medical University.

\section{Consent for publication}

Consents for publication were obtained from all participants.

\section{Competing interests}

The authors declare no competing interests.

\section{Funding}

This study was supported by grants from the National Natural Science Foundation of China (81500007), Natural Science Foundation of Guangdong Province (2019A1515011713),the Medical Science Foundation of Guangdong Province (B2018014), the Science and Technology Project of Dongguan (2017507150054 Research Foundation of Guangdong Medical University for Ph.D. Staff (B2019038) and the Key Cultivation Project of Guangdong Medical University (GDMUZ2019001) and Guangdong Medical University Student Innovation Experiment Project(GDMU2019132, ZZDC001, ZYDG002).Research Foundation of Guangdong Medical University 『M2017012『GDMUM2020006『 


\section{Author Contributions}

J.C.Z, W.Q.Y, J,H, and Z.Y.Y conceived, designed and supervised the overall study. J,H, and Z.Y.Y, Y.Y.L collected and verified the mycobacterial strains. X.X.C, Y.F.L, B.H.L, S.B.Z, Z.P.C, H.L.Z, and X.Y.Z processed the samples and performed the experiments. Z.Z T.C J.H and Z.Y.Y analysed the data. J,H, Z.Y.Y, W.J.W and J.C.Z wrote the paper. All authors read and approved the final manuscript.

\section{Availability of data and material}

The authors confirm that the data supporting the findings of this study are available within the article.

\section{References}

1. Ye J. Roles of regulated intramembrane proteolysis in virus infection and antiviral immunity. Biochim Biophys Acta. 2013;1828:2926-2932.

2. Wilkins T, Sequoia J. Probiotics for Gastrointestinal Conditions: A Summary of the Evidence. Am Fam Physician. 2017; 96:170-178.

3. Shokryazdan P, Jahromi MF, Liang JB, Ho Probiotics: From Isolation to Application. J Am Coll Nutr. 2017;36:666-676.

4. SunZ, Sun X, Li Using probiotics for type 2 diabetes mellitus intervention: Advances, questions, and potential. Crit Rev Food Sci Nutr. 2020;60:670-683.

5. Sivamaruthi BS, Kesika P, Chaiyasut Effect of Probiotics Supplementations on Health Status of Athletes. Int J Environ Res Public Health. $2019 ; 16: 4469$.

6. Yu A, Li The Potential Role of Probiotics in Cancer Prevention and Treatment.Nutr Cancer. 2016;68:535-44.

7. Dimidi E, Scott SM, WhelanProbiotics and constipation: mechanisms of action, evidence for effectiveness and utilisation by patients and healthcare professionals.Proc Nutr Soc. 2020;79:147-157.

8. Smriti Sharma, Seema Puri, Anura V Kurpad.Potential of Probiotics in Hypercholesterolemia: A Review of In Vitro and In Vivo Findings.Altern Ther Health Med . 2018;24:36-43.

9. Kruis W, Chrubasik S, Boehm S, et al. A double-blind placebo-controlled trial to study therapeutic effects of probiotic Escherichia coli Nissle 1917 in subgroups of patients with irritable bowel syndrome. Int J Colorectal Dis. 2012, 27: 467-474.

10. Goel A, Mittal A, Evstatiev R, et al. In vivo effects of mesalazine or E. coli Nissle 1917 on microsatellite instability in ulcerative colitis. Aliment Pharmacol Ther. 2009, 30: 634-642.

11. Kruis W, Fric P, Pokrotnieks J, et al. Maintaining remission of ulcerative colitis with the probiotic Escherichia coli Nissle 1917 is as effective as with standard mesalazine. Gut.2004, 53: 1617-1623.

12. SHEN Zhaohua, ZHU Changxin, QUAN Yongsheng, et al. Relationship between intestinal microbiota and ulcerative colitis: Mechanisms and clinical application of probiotics and fecal microbiota transplantation. World Journal of Gastroenterology. 2018;24: 5-14.

13. Chen CL, Hsu PY, Pan TM. Therapeutic effects of Lactobacillus paracasei subsp. paracasei NTU 101 powder on dextran sulfate sodium-induced colitis in mice. J Food Drug Anal. 2019, 27: 83-92.

14. Sanchez E, Nieto JC, Vidal S, et al. Fermented milk containing Lactobacillus paracasei subsp. paracasei CNCM I-1518 reduces bacterial translocation in rats treated with carbon tetrachloride. Sci Rep, 2017, 7(1): 45712.

15. Author links open overlay panelMarcoToscanoaRobertaDe GrandiaLucaPastorelli,et al.A consumer's guide for probiotics: 10 golden rules for a correct use.Digestive and Liver Disease,2017,49(11):1177-1184.

16. Cesare Cremon,Simone Guglielmetti,Giorgio Gargari,et al.Effect of Lactobacillus paracasei CNCM I-1572 on symptoms, gut microbiota, short chain fatty acids, and immune activation in patients with irritable bowel syndrome: A pilot randomized clinical trial.United European Gastroenterology Journal,2018, 6(4) :604-613.

17. Chiara Ferrario,Valentina Taverniti,Christian Milani,et al.Modulation of fecal Clostridiales bacteria and butyrate by probiotic intervention with Lactobacillus paracasei DG varies among healthy adults.J Nutr. 2014,144(11):1787-1796.

18. Chia-Yuan Chang ,Tzu-Ming Pan.Anticancer and Antimigration Effects of a Combinatorial Treatment of 5-Fluorouracil and Lactobacillus paracasei subsp. paracasei NTU 101 Fermented Skim Milk Extracts on Colorectal Cancer Cells.J Agric Food Chem. 2018,66(22):5549-5555.

19. Huayi Suo, Shanchun Liu, Jian Li, et al.Lactobacillus paracasei ssp paracasei YBJ01 reduced D-galactose-induced oxidation in male Kuming mice.Journal of Dairy Science, 2018, 101(12): 10664-10674.

20. Kaplan GG: The global burden of IBD: from 2015 to 2025. Nat Rev Gastroenterol Hepatol. 2015; 12(12): 720-7.

21. Ross J Porter, Rahul Kalla, Gwo-Tzer Ho.Ulcerative colitis: Recent advances in the understanding of disease pathogenesis.F1000Research 2020, 9:1-13.

22. Cho J, Kim S, Yang DH, et al. Mucosal Immunity Related to FOXP3(+) Regulatory T Cells, Th17 Cells and Cytokines in Pediatric Inflammatory Bowel Disease. J Korean Med Sci, 2018, 33(52): e336.

23. Li Q, Shan Q, Sang X, et al. Total Glycosides of Peony Protects Against Inflammatory Bowel Disease by Regulating IL-23/IL-17 Axis and Th17/Tregs Balance. Am J Chin Med, 2019, 47(1): 177-201.

24. Zou H, He T, Chen X. Tetrandrine inhibits differentiation of proinflammatory subsets of T helper cells but spares de novo differentiation of iTregs cells. Int Immunopharmacol, 2019, 69(4): 307-312.

25. Haribhai D, Chatila TA, Williams CB. Immunotherapy with iTregs and nTregs Cells in a Murine Model of Inflammatory Bowel Disease. Methods Mol Biol, 2016, 1422(6): 197-211. 
26. Jang SW, Hwang SS, Kim HS, et al. Homeobox protein Hhex negatively regulates Tregs cells by inhibiting Foxp3 expression and function. Proc Natl Acad Sci U S A, 2019, 116(51): 25790-25799.

27. Li L, Shi QG, Lin F, et al. Cytokine IL-6 is required in Citrobacter rodentium infection-induced intestinal Th17 responses and promotes IL-22 expression in inflammatory bowel disease. Mol Med Rep, 2014, 9(3): 831-836.

28. Friedrich M, Diegelmann J, Schauber J, et al. Intestinal neuroendocrine cells and goblet cells are mediators of IL-17A-amplified epithelial IL-17C production in human inflammatory bowel disease. Mucosal Immunol, 2015, 8(4): 943-958.

\section{Figures}

A

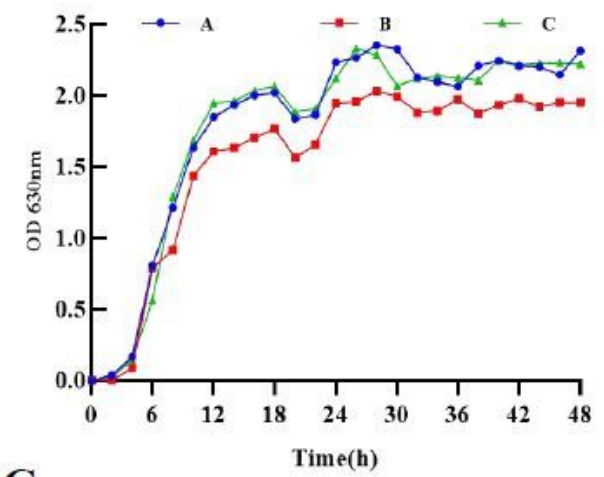

C

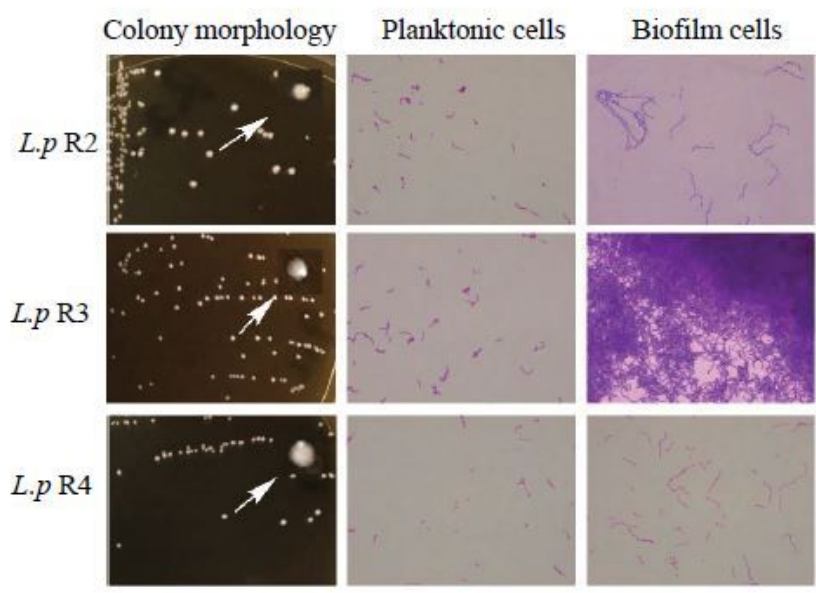

B

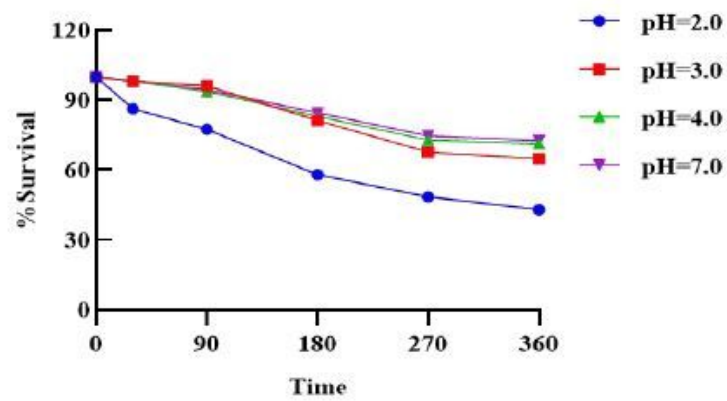

D

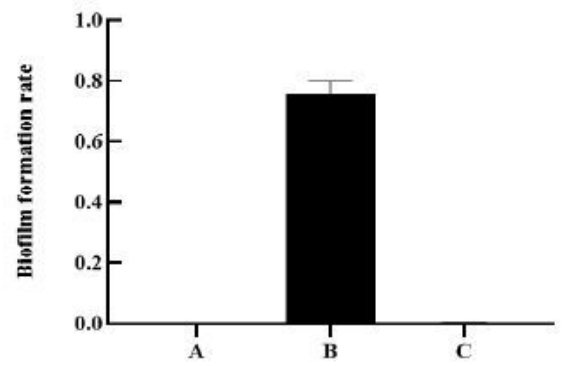

\section{Figure 1}

Biological properties of L.p R3 strain. (A)Growth curves of L.pR2, R3 and R4 strains were detetcted. (B) Gastrointestinal tract tolerance of L.p R3 strain were examined. (C and D) Biofilm formation ability of L. p R2, R3 and R4 strains were measured. 
A

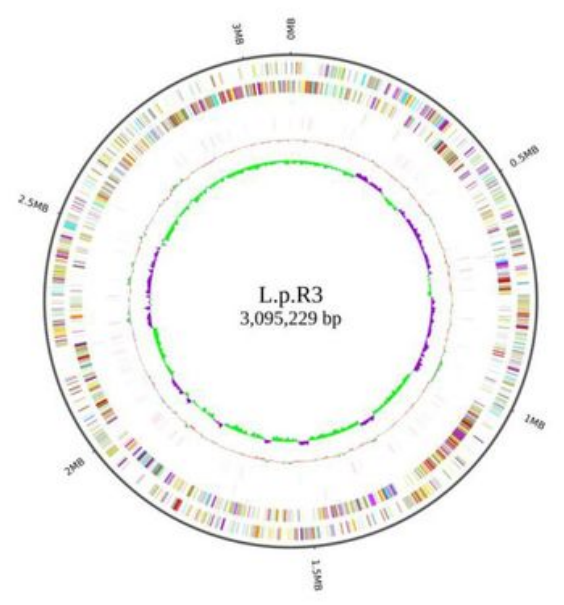

B

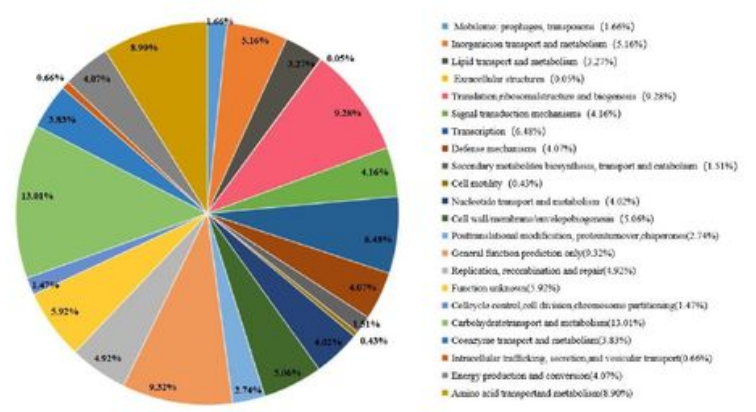

Figure 2

whole genome sequencing analysis of L.p R3 strain. (A)The genome cycle graph of L.p R3. (B)COG Function Classification

A

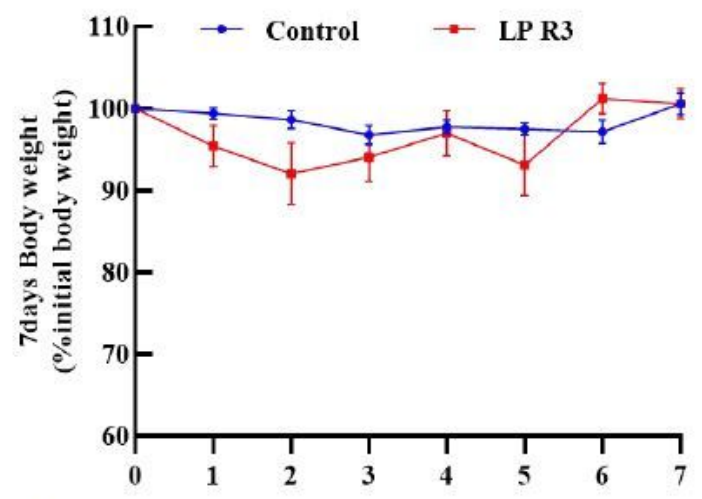

C

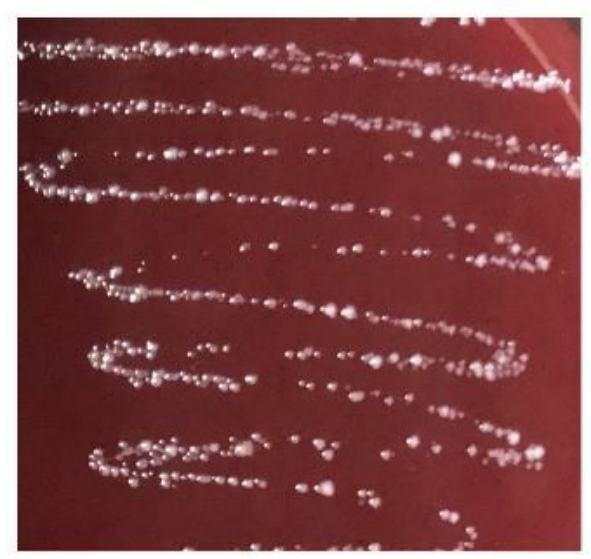

B

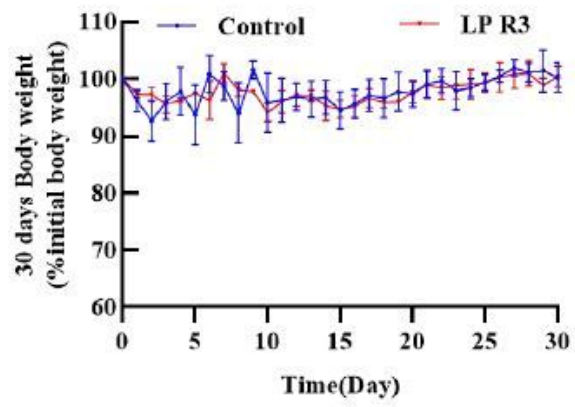

D
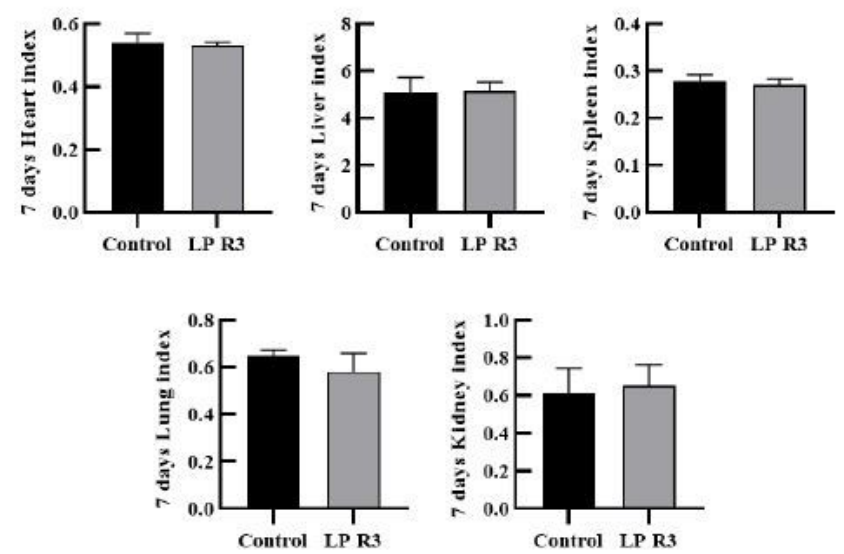
Safety evaluation of L.p R3 (A) Weight changes in mice fed for 7 days. (B)Weight changes in mice fed for 30 days. (C)Experimental results of hemolysis. (D)Organ index of mice.

$\mathbf{A}$

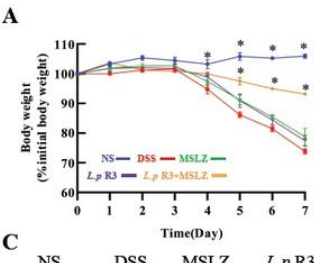

C

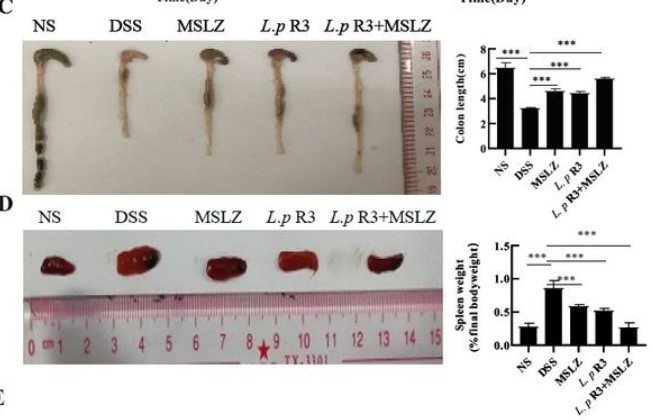

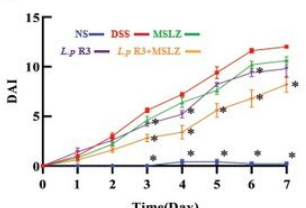

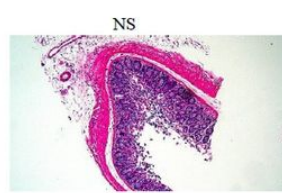

L.p $\mathrm{R} 3$

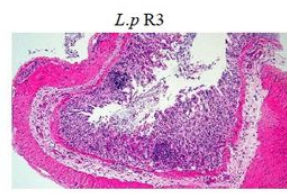

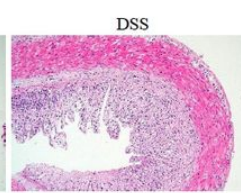

L.p R3+MSLZ

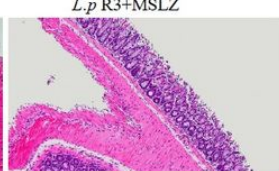

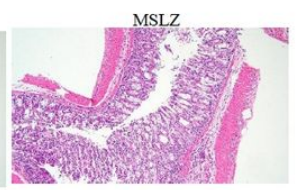

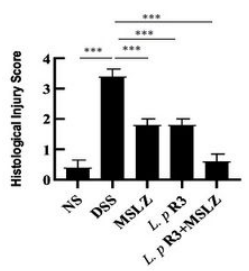

Figure 4

L.p R3 strain ameliorates the general symptoms of murine colitis (A) Percentage change in body weight of mice after induction of colitis.(B) Daily disease activity index (DAl) of mice after induction of colitis.(C) Representative images showing the colon lengths on days 14. (D) Representative images of the spleen and SI on days 14. (E)Histological analysis of the severity of colon sections in DSS-induced colitis mouse model; Colon tissue was paraffin-embedded and stained with hematoxylin and eosin. Cellular infiltration, crypt distortion, and goblet cell loss were analyzed on days 14 . H\&E stain scale: $100 \mathrm{~mm}$. Values are means \pm SEMs $(n=6)$. Statistical analysis was performed by two-way ANOVA with a NewmaneKeuls post-hoc test.*P $<0.05$, compared with DSS group. 
A
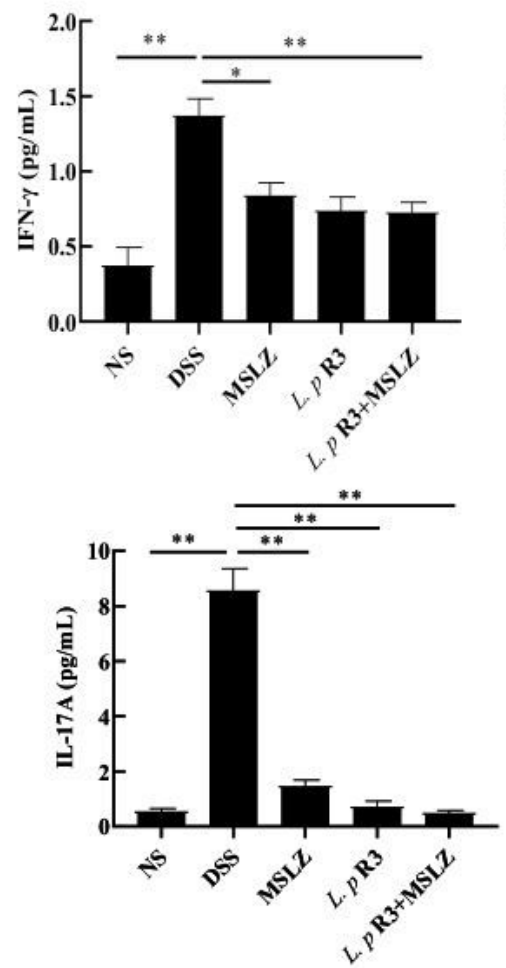

B
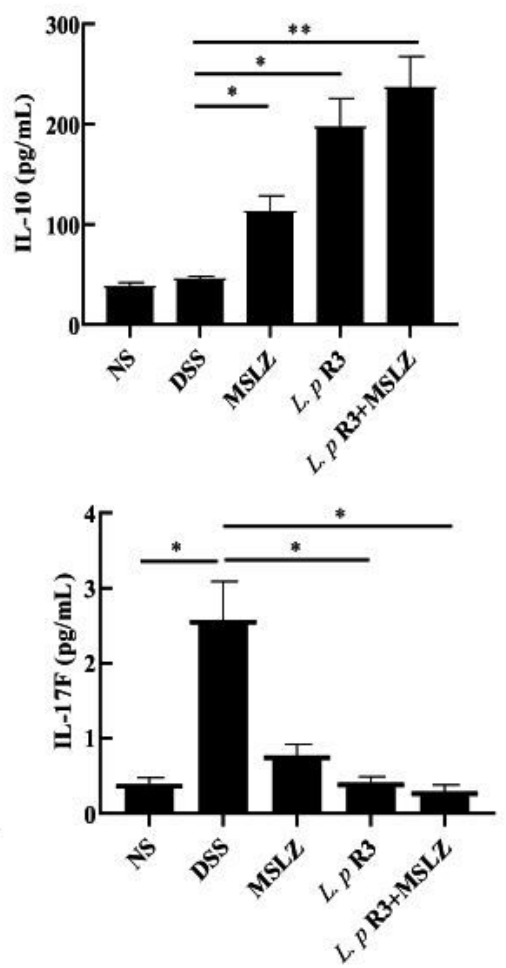

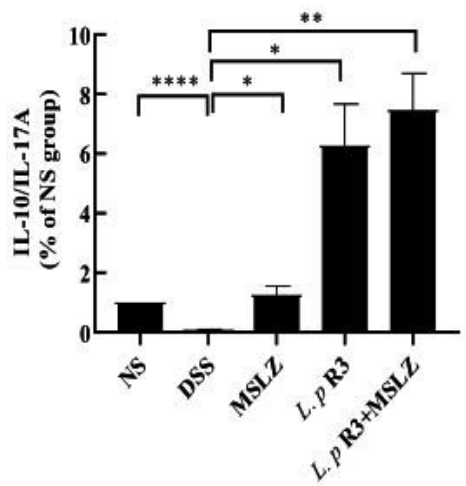

Figure 5

Detection of serum immune cytokines by liquid-phase chip method. (A)The contents of IFN- $y$, IL-17A, IL-17F and IL-10 in peripheral serum.(B)IL-17A/IL-10 in peripheral serum of each group mice.Statistical analysis was performed by two-way ANOVA with a NewmaneKeuls post-hoc test.*P $<0.05$, compared with DSS group. 


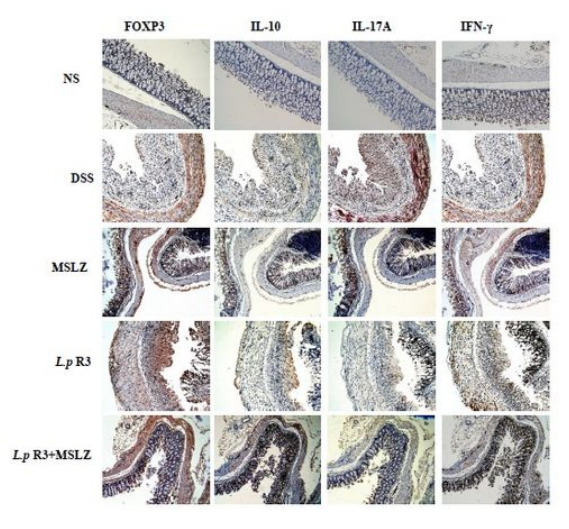

\section{|IIII) IIII

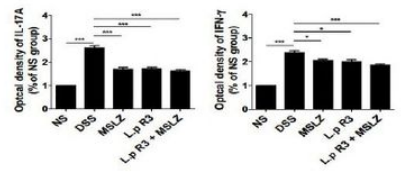

C

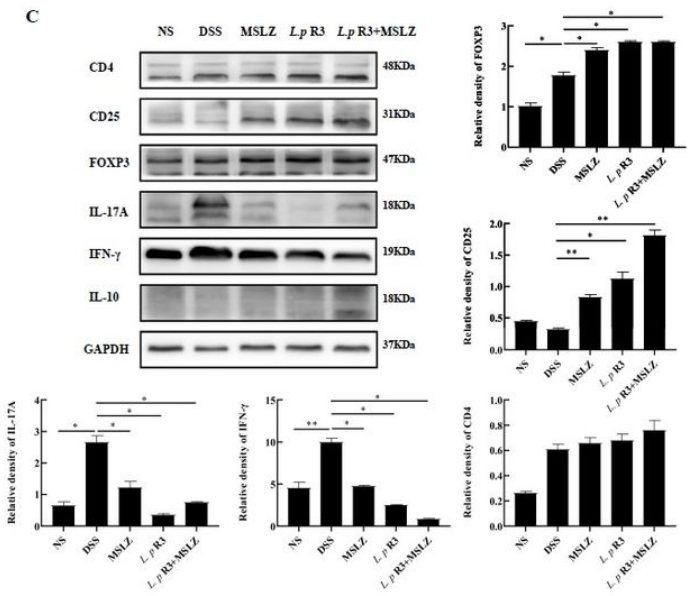

Figure 6

L.p R3 attenuates colonic inflammatory responses but induces Treg responses in DSS-induced experimental murine colitis. (A)Foxp3, IL-10, IL-17A and IFNYwere detected in the colon by the immunohistochemical method. (B)Positive score of colon Foxp3, IL-10, IL-17A and IFN-ypathology in each group. (C) Release of CD4, CD25, FOXP3, IL-17A, IFN-y, IL-10 proteins in the colonic tissues was assessed by immunoblotting and the realative density of CD4, CD25, FOXP3, IL-17A, IFN-yproteins in the colonic tissues was assayed by Image-Pro Plus ${ }^{\star}, \mathrm{P}<0.05 ; * \star, P<0.01 ; * \star \star, P<0.001$. The results are representative of at least three independent experiments. 